\title{
Partial Breast Reconstruction Using Various Oncoplastic Techniques for Centrally Located Breast Cancer
}

\author{
Hyo Chun Park ${ }^{1}$, Hong Yeul Kim ${ }^{1}$, Min Chul Kim², Jeong Woo Lee ${ }^{2}$, Ho Yun Chung ${ }^{2}$, \\ Byung Chae $\mathrm{Cho}^{2}$, Ho Yong Park ${ }^{3}$, Jung Dug Yang ${ }^{2}$ \\ ${ }^{1}$ Department of Plastic and Reconstructive Surgery, Daegu Fatima Hospital, Daegu; ${ }^{2}$ Department of Plastic and Reconstructive Surgery, \\ Kyungpook National University School of Medicine, Daegu; ${ }^{3}$ Department of Surgery, Kyungpook National University School of Medicine, \\ Daegu, Korea
}

Background As the breast cancer incidence has increased, breast-conserving surgery has replaced total mastectomy as the predominant procedure. However, centrally located breast cancers pose significant challenges to successful breast-conserving surgeries. Therefore, we performed partial mastectomy and oncoplastic procedures on centrally located breast cancer as a means of partial breast reconstruction. The authors examined and evaluated the functional and aesthetic usefulness of this reconstruction method.

Methods From January 2007 to June 2011, 35 patients with centrally located breast cancers who underwent various oncoplastic procedures based on the breast size and resection volume. The oncoplastic procedures performed included volume displacement surgical techniques such as purse-string suture, linear suture, and reduction mammaplasty. Other oncoplastic procedures included volume replacement procedures with an adipofascial, thoracoepigastric, intercostal artery perforator, thoracodorsal artery perforator, or latissimus dorsi flap.

Results Mean patient age was 49 years, and mean follow-up period was 11 months. In cases of small to moderate-sized breasts and resection volumes $<50 \mathrm{~g}$, volume displacement procedures were performed. In cases of resection volumes $>50 \mathrm{~g}$, volume replacement procedures were performed. In cases of larger breasts and smaller resection volumes, glandular reshaping was performed. Finally, in cases of larger breasts and larger resection volumes, reduction mammaplasty was performed. This reconstruction method also elicits a high patient satisfaction rate with no significant complications.

Conclusions In centrally located breast cancer, oncoplastic surgery considering breast size and resection volume is safe and provides appropriate aesthetic outcomes. Therefore, our method is advisable for breast cancer patients who elect to conserve their breasts and retain a natural breast shape.

Keywords Breast neoplasms / Surgical flaps / Mammaplasty
Correspondence: Jung Dug Yang Department of Plastic and Reconstructive Surgery, Kyungpook National University School of Medicine, 130 Dongdeok-ro, Jung-gu, Daegu 700-421, Korea Tel: +82-53-200-5685 Fax: +82-53-425-3879 E-mail: lambyang@knu.ac.kr

No potential conflict of interest relevant to this article was reported. 


\section{INTRODUCTION}

Recently, the incidence of breast cancer in Korea has increased, and since 2001, breast cancer has become the most prevalent type of cancer among Korean women. With the development of breast cancer diagnostic techniques and the universalization of regular check-ups, early-stage discoveries of very small breast tumors have become frequent. Consequently, breast-conserving surgical procedures via partial mastectomy are performed more frequently than total mastectomies in order to minimize breast deformation.

However, breast-conserving surgery is avoided in cases of centrally located breast cancers, given the possibility of multifocality, multicentricity, direct invasion of the nipple-areolar complex, and aesthetic revulsion arising from the possible removal of the nipple-areolar complex [1-5].

Despite these challenges, studies and observations concerning centrally located breast cancer patients have consistently conformed to the trend of breast-conserving treatment. Simmons et al. [6] stated that there was no statistical difference in the recurrence rates with breast-conserving surgery and total mastectomy. Horiguchi et al. [7] also found that radiation treatment after breast-conserving surgery yielded optimal results for early-stage cancer patients with centrally located breast cancers. Huemer et al. [8] reported that various oncoplastic procedures could provide satisfactory results for centrally located breast cancers. The present study also yielded positive results by applying oncoplastic procedures to centrally located breast cancers.

Given this background, for centrally located breast cancer, breast-conserving surgery and partial breast reconstruction might be applied. We applied various oncoplastic procedures according to breast size and resection volume, and obtained satisfactory results. We expect our method to become a guideline for the oncoplastic surgery of centrally located breast cancer.

\section{METHODS}

\section{Subjects}

The subjects of this study were 35 centrally located breast cancer patients who underwent breast reconstruction procedures between January 2007 and June 2011. The procedures included a partial breast resection, performed by the surgical department, and oncoplastic surgery by a plastic surgeon immediately afterward. According to the classification of Simmons et al. [6], Haffty et al. [9], and Fowble et al. [10], a centrally located breast cancer was defined as a cancer located within $2 \mathrm{~cm}$ of the areola boundary.

\section{Surgical methods}

A complete nipple-areolar complex resection was included in the procedure to obtain a tumor-free margin. The free margin criterion was defined as the absence of tumor cells in regions that were at least $1 \mathrm{~mm}$ from the resection margin according to a pathology examination of postoperative permanent sections. In cases of possible preoperative metastasis to the lymph nodes or the discovery of cancerous cells during a frozen section biopsy of axillary sentinel lymph nodes, lymph node resections were also performed.

Various oncoplastic procedures were performed on the basis of the ratio of the breast size and the tumor resection volume (Fig. $1)$. In cases of small-to-moderate-sized breasts and small resection volumes ( $<50 \mathrm{~g}$, small defect), volume displacement procedures such as purse-string suture or linear suture were performed; in cases of moderate resection volumes $(<150 \mathrm{~g}$, moderate defect), volume replacement procedures using an adipofascial, thoracoepigastric, or intercostal artery perforator (ICAP), or a thoracodorsal artery perforator (TDAP) flap were performed; and in cases of large resection volumes (>150 g, large defect), volume replacement procedures using a latissimus dorsi (LD) flap were performed. In addition, in cases of large breasts and small resection volumes, glandular reshaping was performed; in cases of large breasts and large resection volumes, reduction mammaplasty was performed. Factors such as skin defect size, amount of required volume, position of the defect, and symmetry of breasts were also considered.

In the case of a pedicled flap, a supramuscular tunnel was created between the recipient site and the defect. The flap passed through this tunnel, and the pedicle could be located in this plane. By skeletonization, as much of the soft tissue around the

Fig. 1. Oncoplastic techniques for centrally located breast cancer

Flowchart of oncoplastic techniques for the treatment of centrally located breast cancer in small-to-moderate-sized breasts. T-E, thoracoepigastric; ICAP, intercostal artery perforator; TDAP, thoracodorsal artery perforator; LD, latissimus dorsi.

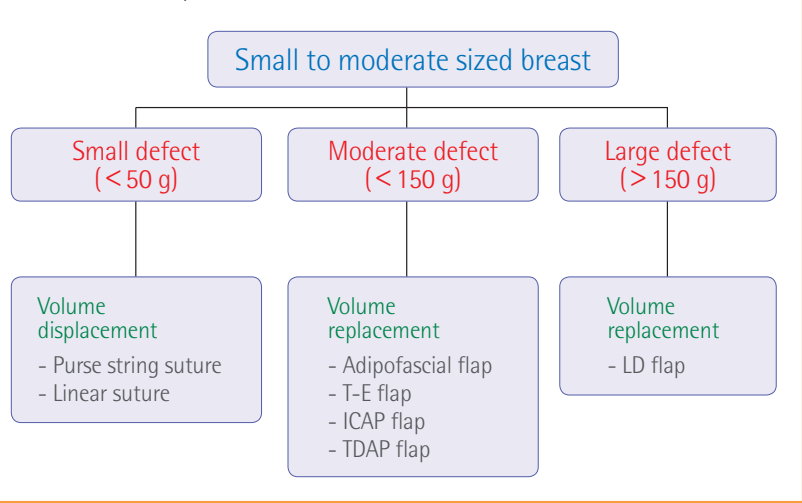


Table 1. Modified Michigan breast reconstruction outcomes survey

General satisfaction

1. Knowing what I know today, I would definitely choose to undergo breast reconstruction.

2. Knowing what I know today, I would definitely choose to undergo the same type of reconstruction.

3. Overall, I am satisfied with my reconstruction.

4. I would recommend the type of reconstructive procedure that I underwent to a friend.

5. I felt that I received sufficient information about my reconstruction options to make an informed decision from among several procedures.

Aesthetic satisfaction

1. My breasts are the same size and shape.

2. My reconstructed breast(s) feel soft to the touch.

Modified from Alderman et al. Plast Reconstr Surg 2000;106:769-76, with permission from the American Society of Plastic Surgeons [11].

Fig. 2. General and aesthetic satisfaction by patients

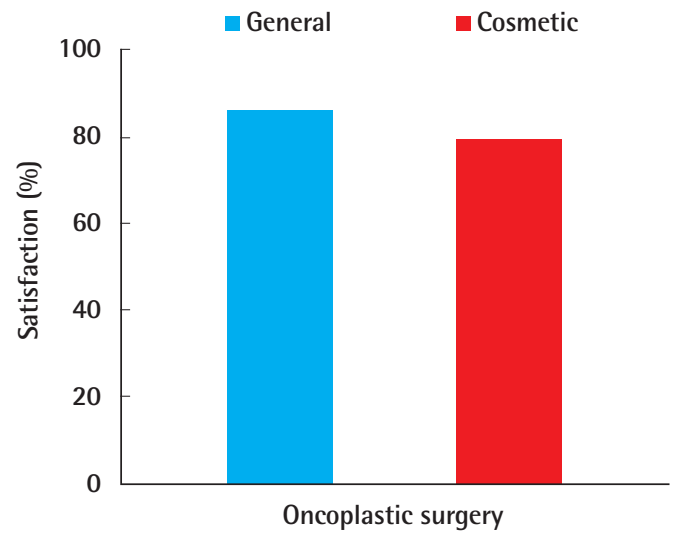

pedicle as possible was removed and postoperative bulging was minimized. We also avoided compression of the flap pedicle by ensuring sufficient space. This could prevent a postoperative vascular compromising event.

Nipple reconstruction was performed after the end of postoperative radiation therapy and chemotherapy, which were performed an average of 6 months after surgery. We performed nipple reconstruction with a Hammond flap or a C-V flap, and approximately 2 months later, tattooing was performed for areolar pigmentation.

Six months after surgery, the Modified Michigan Breast Reconstruction Outcome Survey was administered to gauge the patients' aesthetic and overall satisfaction (Table 1) [11]. Five types of questions were used to evaluate the overall satisfaction level, whereas two types of questions were used to evaluate aesthetic satisfaction. Each question was assigned a score from 1 to 5 , with 1 indicating "highly unsatisfactory" and 5 indicating "highly satisfactory." This scale was later reclassified as unsatisfactory (1-3 points) and satisfactory (4-5 points) (Fig. 2). Ad-
Fig. 3. Evaluation of breast shape and symmetry

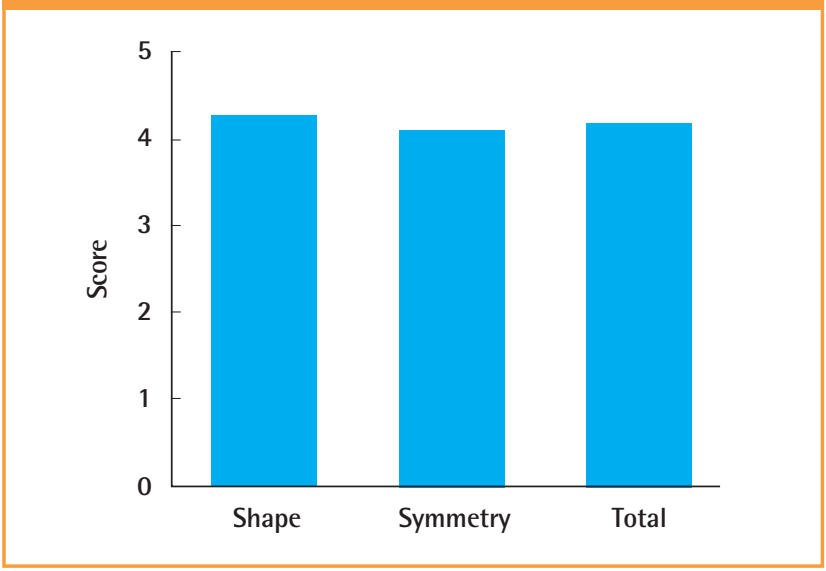

ditionally, postoperative photographs were shown to three surgeons who aesthetically evaluated the breast shape and symmetry; these were also rated from 1 point (unsatisfactory) to 5 points (very satisfactory) (Fig. 3).

\section{RESULTS}

Breast reconstruction employing various oncoplastic procedures was performed on 35 patients after partial breast resection. The patient ages ranged from 28 to 64 years, with a mean value of 49 years, and the mean observation period was 11 months. The patients' cancer stages were determined to range from stage 0 to stage II, according to the tumor-node-metastasis (TNM) staging system of the American Joint Committee on Cancer (AJCC). There were 24 cases of invasive ductal tumors (IDC), 10 cases of ductal tumors in situ (DCIS), and 1 case of a phyllodes tumor. The mean tumor mass was $89.5 \mathrm{~g}$ (range, 21-284 g), and the mean distance between the nipple and the tumor, measured during the final pathology examination, was $1.5 \mathrm{~cm}$ (range, $0.2-2$ $\mathrm{cm})$. Invasive tumors were confirmed in 7 (20\%) patients via sentinel node biopsy; these patients required axillary lymph node dissection. After resection, 34 patients received radiation treatment, 19 patients received chemotherapy, and 23 patients received hormone therapy. Because the single patient with a phyllodes tumor did not postoperatively exhibit a tumor in the resection margins according to the pathological findings, this patient was monitored without any further treatment during the follow-up period.

In cases of small-to-moderate-sized breasts, volume displacement procedures including 8 purse-string sutures and 7 linear sutures were performed. Volume replacement procedures including 1 adipofascial flap, 2 thoracoepigastric flaps, 7 ICAP flaps, 1 TDAP flap, and 3 LD flaps were performed. In cases of large breasts, 1 vertical incision, 3 glandular reshaping, and 2 inverted 
Table 2. Characteristics of the 35 patients who were treated for centrally located breast cancer

\begin{tabular}{|c|c|c|c|c|c|c|c|}
\hline Patient & $\begin{array}{l}\text { Age } \\
(y r)\end{array}$ & $\begin{array}{l}\text { Type of } \\
\text { tumor }\end{array}$ & $\begin{array}{l}\text { Distance from } \\
\text { nipple }(\mathrm{cm})\end{array}$ & $\begin{array}{l}\text { Weight of } \\
\text { tumor }(\mathrm{g})\end{array}$ & $\begin{array}{l}\text { TNM } \\
\text { stage }\end{array}$ & $\begin{array}{l}\text { SLN } \\
\text { status }\end{array}$ & $\begin{array}{l}\text { Surgical } \\
\text { technique }\end{array}$ \\
\hline 1 & 28 & IDC & 2 & 213 & $\| \mathrm{A}$ & Positive & Latissimus dorsi flap \\
\hline 2 & 50 & IDC & 0.3 & 42 & I & Negative & Linear suture \\
\hline 3 & 42 & DCIS & 0.6 & 236 & 0 & Negative & Reduction (inverted T) \\
\hline 4 & 57 & DCIS & 1.9 & 124 & I & Negative & Reduction (vertical) \\
\hline 5 & 52 & DCIS & 0.2 & 21 & I & Negative & Linear suture \\
\hline 6 & 39 & IDC & 1.8 & 47 & I & Negative & Purse-string suture \\
\hline 7 & 51 & DCIS & 1.9 & 80 & $\| A$ & Negative & Purse-string suture \\
\hline 8 & 39 & IDC & 1.8 & 84 & $\| A$ & Negative & Glandular reshaping \\
\hline 9 & 48 & DCIS & 1.5 & 92 & 0 & Negative & Glandular reshaping \\
\hline 10 & 52 & $\mathrm{IDC}$ & 1.7 & 61 & I & Negative & Purse-string suture \\
\hline 11 & 60 & IDC & 1.1 & 44 & $\| \mathrm{A}$ & Negative & Linear suture \\
\hline 12 & 36 & DCIS & 2 & 206 & 0 & Negative & Latissimus dorsi flap \\
\hline 13 & 61 & IDC & 1.8 & 95 & I & Negative & Thoracoepigastric flap \\
\hline 14 & 54 & IDC & 2 & 73 & $\| A$ & Negative & Thoracoepigastric flap \\
\hline 15 & 47 & DCIS & 1.4 & 31 & 1 & Negative & Linear suture \\
\hline 16 & 48 & IDC & 2 & 115 & I & Positive & TDAP flap \\
\hline 17 & 44 & Phyllodes tumor & 1.5 & 60 & $\| \mathrm{A}$ & - & Purse-string suture \\
\hline 18 & 50 & IDC & 1.6 & 29 & $\| A$ & Negative & Linear suture \\
\hline 19 & 45 & IDC & 1.9 & 64 & I & Positive & Glandular reshaping \\
\hline 20 & 64 & DCIS & 0.8 & 39 & I & - & Purse-string suture \\
\hline 21 & 46 & IDC & 1.6 & 284 & $\| \mathrm{A}$ & Negative & Reduction (inverted T) \\
\hline 22 & 61 & IDC & 0.5 & 62 & 1 & Positive & Purse-string suture \\
\hline 23 & 49 & IDC & 1.8 & 69 & I & Negative & Purse-string suture \\
\hline 24 & 51 & IDC & 1.7 & 49 & I & Positive & Linear suture \\
\hline 25 & 56 & IDC & 1.4 & 156 & I & Positive & Latissimus dorsi flap \\
\hline 26 & 58 & IDC & 1.6 & 88 & I & Negative & Adipofascial flap \\
\hline 27 & 39 & IDC & 1.3 & 51 & I & Negative & Linear suture \\
\hline 28 & 49 & DCIS & 1.7 & 92 & 1 & Negative & ICAP flap \\
\hline 29 & 51 & IDC & 1.9 & 52 & I & Negative & Purse-string suture \\
\hline 30 & 56 & IDC & 0.8 & 77 & I & Negative & ICAP flap \\
\hline 31 & 53 & IDC & 1.9 & 65 & I & Negative & ICAP flap \\
\hline 32 & 44 & IDC & 1.4 & 82 & $\| \mathrm{A}$ & Negative & ICAP flap \\
\hline 33 & 45 & IDC & 1.6 & 88 & I & Positive & ICAP flap \\
\hline 34 & 46 & IDC & 1.3 & 71 & $\| A$ & Negative & ICAP flap \\
\hline 35 & 56 & DCIS & 1.8 & 92 & I & Negative & ICAP flap \\
\hline
\end{tabular}

T-scar breast reduction procedures were performed (Table 2).

Regarding the postoperative complications, a single case of fat necrosis was reported after a TDAP flap procedure, and 2 cases of congestion were found after ICAP flap procedures. Additionally, 1 case of wound dehiscence was found during a pursestring suture procedure. All of the above complications disappeared after the conserving treatments were completed. No other flap loss or wound infection was observed.

Of the patients, $86 \%$ and $79 \%$ reported a score of 4 or 5 , indicating "satisfaction" in the overall satisfaction and aesthetic satisfaction surveys, respectively (Fig. 2). In the survey of surgeons regarding the aesthetic evaluation, the breast shape and symmetry received mean scores of 4.24 points and 4.08 points, yielding a mean score of 4.16 points and indicating that the overall results were satisfactory (Fig. 3).

\section{Case 1}

A 44-year-old patient presented to the outpatient clinic in our department with a 3-week history of a palpable mass in the left breast. Ultrasonography revealed a $1.2-\mathrm{cm}$ tumor mass at the 3 o'clock position, within $1 \mathrm{~cm}$ of the nipple. Follow-up core needle biopsy confirmed the presence of a phyllodes tumor. Therefore, a partial excision $(60 \mathrm{~g})$ and an oncoplastic purse-string suture procedure were performed. The patient was postoperatively monitored for 10 months and did not exhibit any notable complications or tumor recurrence (Fig. 4).

\section{Case 2}

A 53-year-old patient presented with a microcalcification in the right breast during an early check-up. Ultrasonography revealed a $2.5-\mathrm{cm}$ tumor mass at the 10 o'clock position, within $2 \mathrm{~cm}$ of 


\section{Fig. 4. A case of purse-string suture}

A 44-year-old woman with a phyllodes tumor in the left central breast. (A) Preoperative view of the patient. (B) Intraoperative view of partial mastectomy (weight of tumor, $60 \mathrm{~g}$ ). (C) 10-month postoperative outcome.
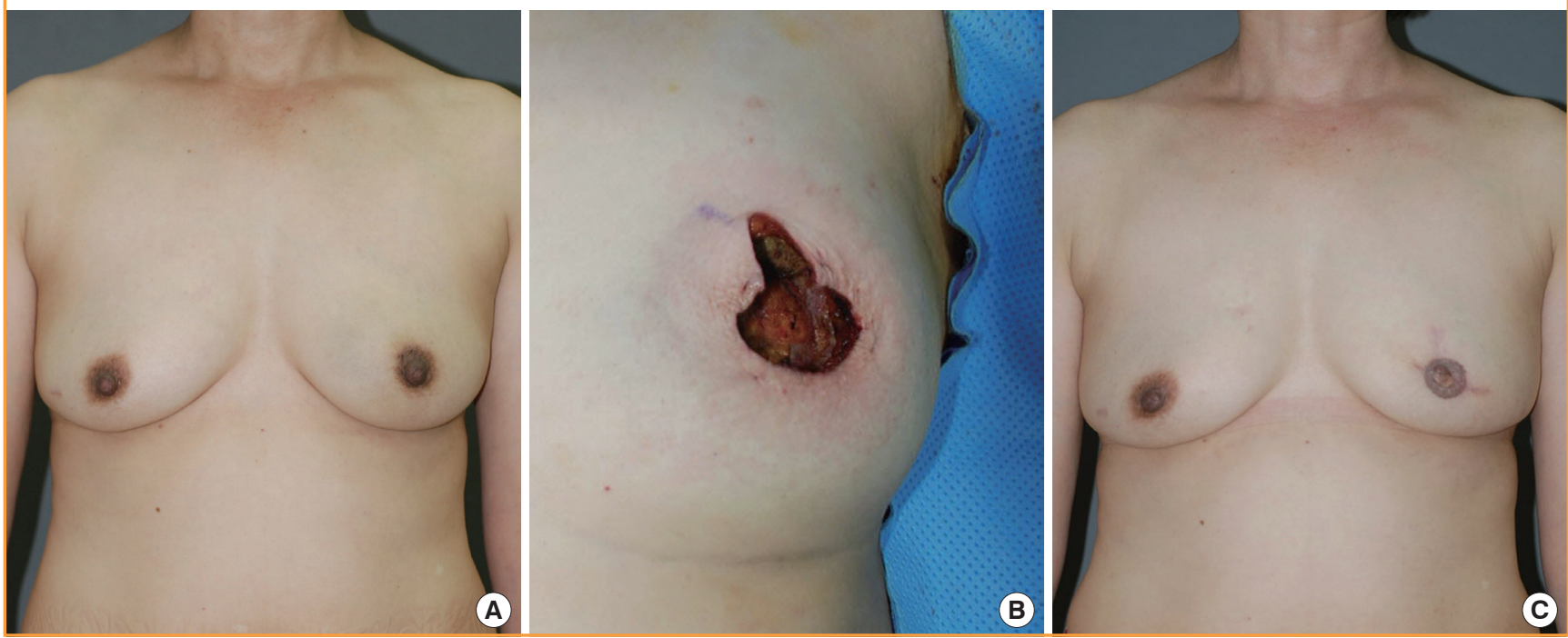

Fig. 5. A case of intercostal artery perforator flap

A 53-year-old woman with invasive ductal carcinoma in the left central breast. (A) Preoperative view of the patient. (B) Intraoperative view of elevated intercostal artery perforator flap after partial mastectomy (weight of tumor, $75 \mathrm{~g}$ ). (C) 19-month postoperative outcome.

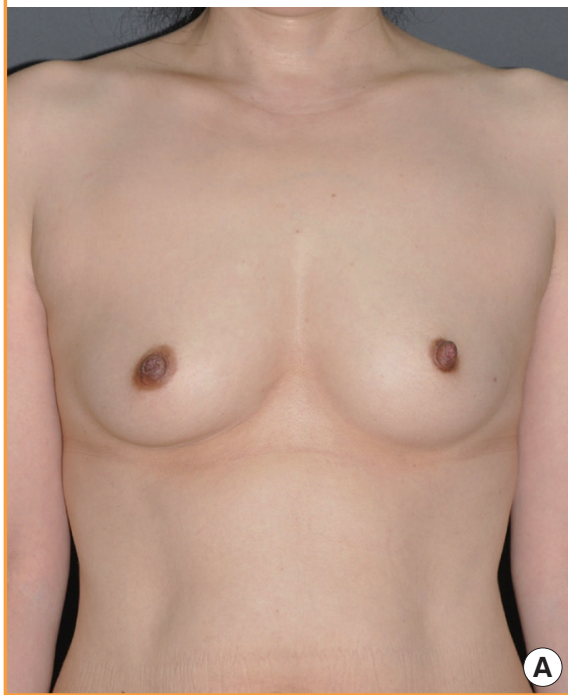

the nipple. Follow-up core needle biopsy confirmed the presence of an IDC. Consequently, a partial mastectomy ( $75 \mathrm{~g})$ was performed, immediately followed by an oncoplastic ICAP flap procedure. The patient was postoperatively monitored for 6 months and did not exhibit any notable complications or tumor recurrence (Fig. 5).

\section{Case 3}

A 36-year-old patient presented to the outpatient clinic of our department with a 1-month history of a palpable mass in the right breast. Ultrasonography revealed a 3 -cm tumor mass at the
10 o'clock position, within $1.5 \mathrm{~cm}$ of the nipple. Follow-up core needle biopsy confirmed the presence of an IDC. Therefore, a partial mastectomy $(206 \mathrm{~g})$ was performed, immediately followed by an oncoplastic LD flap procedure. The patient was postoperatively monitored for 30 months and did not exhibit any notable complications or tumor recurrence (Fig. 6).

\section{Case 4}

A 57-year-old patient presented with a microcalcification in the left breast during an early check-up. Ultrasonography revealed a $2.5-\mathrm{cm}$ tumor mass at the 6 o'clock position, within $0.6 \mathrm{~cm}$ of 


\section{Fig. 6. A case of latissimus dorsi (LD) flap}

A 36-year-old woman with ductal carcinoma in situ in the right central breast. (A) Preoperative view of the patient. (B) Intraoperative view of elevated latissimus dorsi flap after partial mastectomy (weight of tumor, $206 \mathrm{~g}$ ). (C) 30-month postoperative outcome.
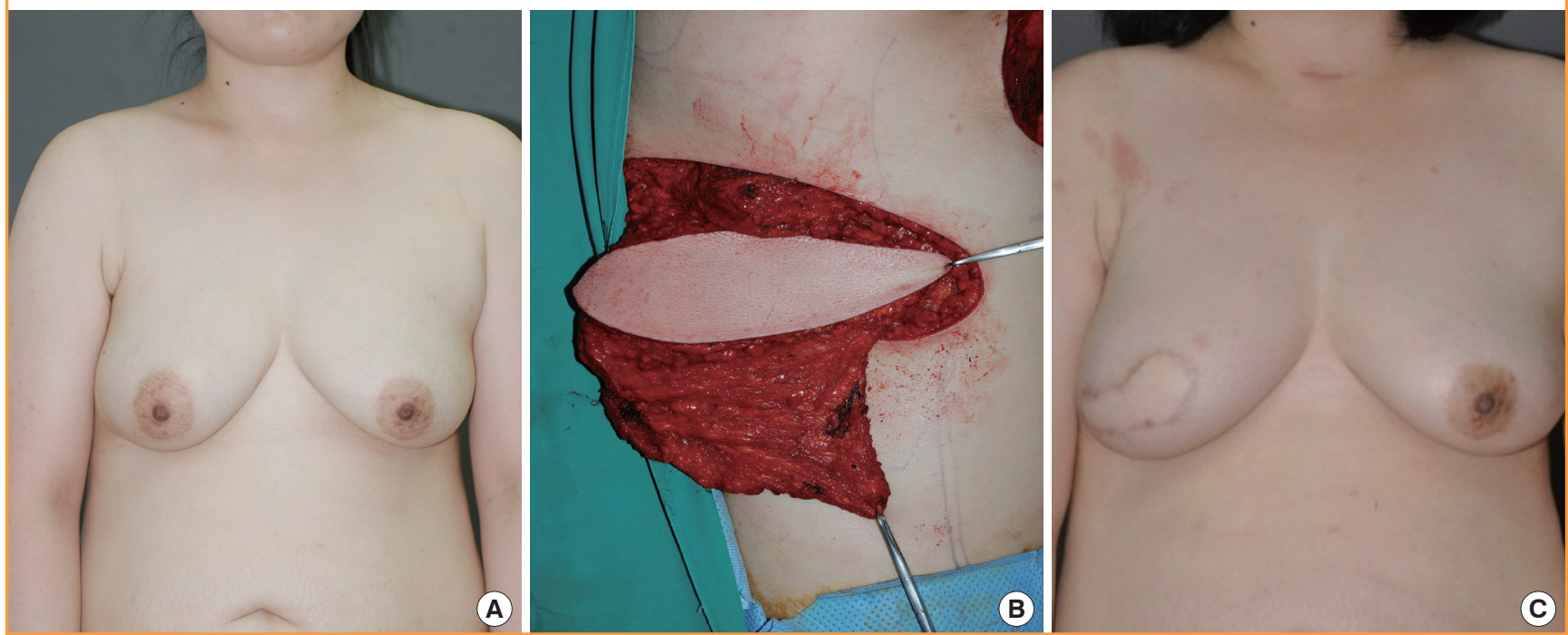

Fig. 7. Case of vertical-type reduction mammaplasty

A 57-year-old woman with ductal carcinoma in situ in the left central breast. (A) Preoperative view of the patient. (B) Immediate postoperative view of vertical-type reduction mammaplasty (in contralateral breast, reduction mammaplasty was performed for symmetrization). (C) 10-month postoperative outcome.
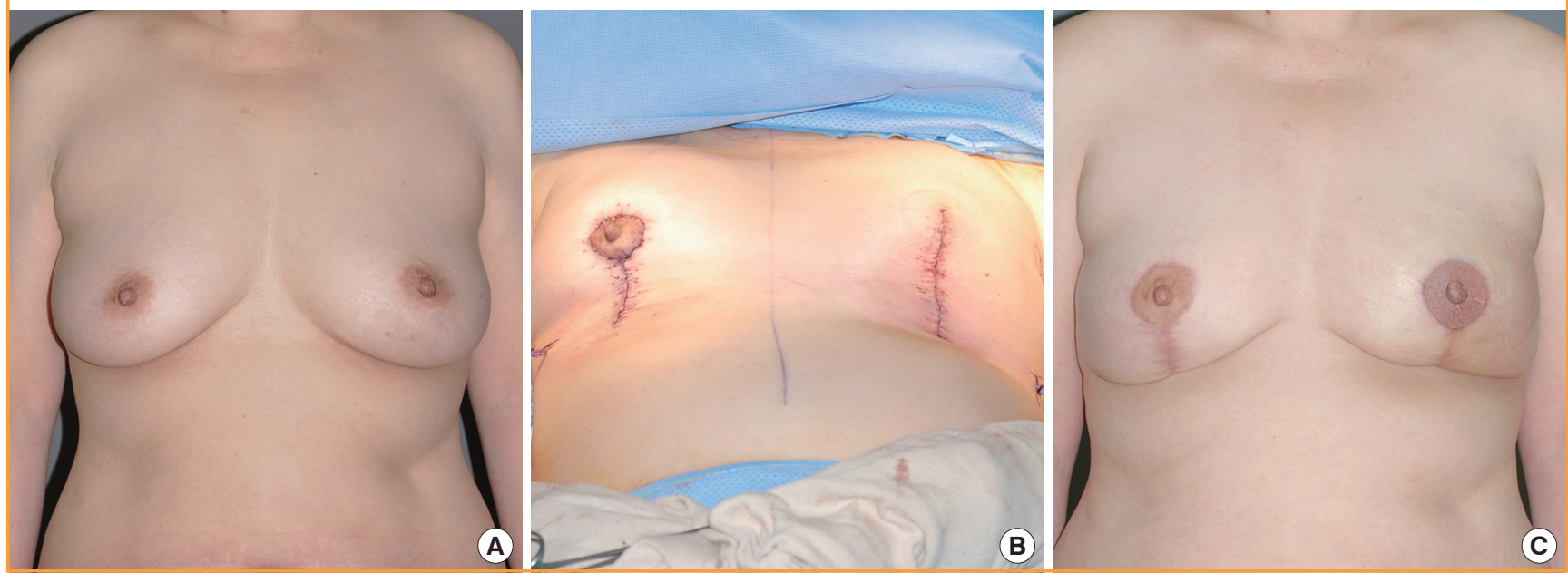

the nipple. Follow-up core needle biopsy confirmed the presence of DCIS. Therefore, a partial mastectomy of the left breast $(124 \mathrm{~g})$ and a vertical reduction mammaplasty were concurrently performed. For this patient, the medial and lateral glandular tissues were dissected and then joined. A vertical reduction mammaplasty $(119 \mathrm{~g})$ was also performed on the non-cancerous right side to retain breast symmetry. The patient was postoperatively monitored for 10 months and did not exhibit any notable complications or tumor recurrence (Fig. 7).

\section{DISCUSSION}

As reported in several studies, it has been confirmed that no significant differences exist in the survival and recurrence rates of patients who undergo radical mastectomy and breast-conserving operations. Additionally, breast-conserving surgery is generally performed for early-stage breast cancers [12,13]. The recent increase in the frequency of early-stage breast cancers in South Korea has resulted in an increase in the number of breast-conserving operations. As a result, the aesthetic aspects of breastconserving surgery have been given an increasing amount of at- 
tention. Accordingly, oncoplastic procedures have been mentioned as a useful alternative to various types of breast reconstruction surgery, as their stable aesthetic effects have been demonstrated in several studies.

Unlike breast cancers in other areas, centrally located breast cancers were conventionally treated via total mastectomy because of the possibility of multifocality, multicentricity, direct invasion of the nipple-areolar complex, and aesthetic revulsion caused by the removal of the nipple-areolar complex [1-5]. However, total mastectomy is difficult for patients to accept, for both psychological and aesthetic reasons. Recent studies have reported that there are no significant differences between total mastectomy and breast-conserving surgery with respect to local recurrence, distant metastasis, survival rate, and aesthetic satisfaction, even in patients with breast cancers located proximal to the nipple.

Simmons et al. [6] reported that the recurrence rate of patients with centrally located breast cancer who underwent breast-conserving surgery was $6.3 \%$, a statistically insignificant rate as compared to the recurrence rate of $4.5 \%$ in patients who underwent radical mastectomy. Fowble et al. [10] reported similar results. Horiguchi et al. [7] also reported that radiotherapy would be the best treatment for patients with early-stage central breast cancer after breast-conserving surgery with nipple resection. Huemer et al. [8] reported that by using various oncoplastic methods for centrally located breast cancer, they obtained satisfactory outcomes in terms of oncological stability and aesthetic aspects.

To treat patients with centrally located breast cancer, we used various types of oncoplastic procedures depending on the ratio of the tumor resection volume to the breast size. In particular, in cases of small-to-moderate-sized breasts and small resection volumes ( $<50 \mathrm{~g}$, small defect), volume displacement procedures such as purse-string suture or linear suture were performed; in cases of moderate resection volumes $(<150 \mathrm{~g}$, moderate defect), volume replacement procedures using an adipofascial, thoracoepigastric, ICAP, or TDAP flap were performed; and in cases of large resection volumes (>150 g, large defect), volume replacement procedures using an $\mathrm{LD}$ flap were performed (Fig. 1). In addition, in cases of large breasts and small resection volumes, glandular reshaping was performed, whereas in cases of large breasts and large resection volumes, reduction mammaplasty was performed.

Adipofascial, thoracoepigastric, and ICAP flaps have the advantages of causing fewer complications and simplifying the surgery, as these methods restore breast defects by employing the fundamental surgical techniques of rotation or transposition near the chest area. Simultaneously, these methods are less invasive and thus, require a shorter hospitalization period. In addi- tion, because the flaps are compositionally similar to the original breast skin, the patient experience is better than that in the case of a TDAP or LD flap. The disadvantages of using TDAP and LD flaps, which are distant flaps, are that the associated operations are not only longer and more difficult but also carry the risk of complications at the donor sites. However, these methods are advantageous in that a sufficient volume of autologous tissue can be used for the reconstruction.

A thoracoepigastric flap uses the intercostal artery as a vascular pedicle, and the surgical procedure is relatively simple. In addition, sufficient skin and subcutaneous tissues are located under the breast to allow the primary suture to be performed at the donor site, even without additional dissection [14].

An ICAP flap is based on the perforator of the intercostal artery and can be classified as a lateral or anterior ICAP flap, according to the location of the pedicles [15]. The pedicles of the lateral intercostal artery are located on the lateral rib segment of the chest, whereas those of the anterior ICAP flap are located on the anterior muscle segment. This flap type is used as a turnover to expand the volume of the lower side of the breast. The flap axis is drawn along the inframammary sulcus so that the resulting scar can be hidden and is therefore a favorable indication for patients with significant amounts of remnant skin under the breast and those with breast ptosis.

Because the TDAP flap does not impair the function of the LD muscle while elevating the adipocutaneous flap, it can shorten the patient's recovery period by reducing the shoulder movement restriction according to the elevation of the LD muscle. This procedure can also reduce complications such as seroma at the donor sites [16]. However, similar to other fasciocutaneous flaps, fibrosis or volume loss might occur as a secondary effect after radiotherapy on the reconstructed breasts; such secondary effects might lead to aesthetically negative results [17]. While monitoring a case in which a TDAP flap was used, we also noticed a depression in the breast caused by the volume loss, as well as fat necrosis after radiotherapy treatment.

In general, fibrosis or volume loss can occur as a secondary effect after radiotherapy. Thus, there could be some volume loss as a result of the abovementioned breast reconstruction method. In our study, radiotherapy was performed for most patients after surgery, and we experienced volume loss in the case of some patients. Considering the volume decrease, breast reconstruction was slightly overcorrected when volume replacement including a perforator flap and an LD flap was performed. Because our follow-up period was relatively short, an ongoing evaluation of the breast volume is needed for an accurate assessment.

Breast reconstruction using the LD was the first breast construction technique to use purely autologous tissue [18]. For 
Caucasians, the use of this technique has become less common because it cannot provide sufficient volume for breast reconstruction. However, for Asians, who have relatively small breasts, this technique can be effectively used for large defects after breast-conserving surgery.

In cases of large breasts accompanied by breast ptosis, reduction mammaplasty can be performed after partial breast resection. The theoretical advantages of a reduction mammaplasty include its usefulness in obtaining aesthetic effects, a reduction in the risk of breast cancer recurrence consequent to the excision of the greatest possible amount of breast tissue, and improved symptoms in the case of large breasts [19]. However, infections and sensory changes in the nipple-areolar complex that can result when this procedure is performed on a normal contralateral breast should be considered.

In cases of the residual presence of the nipple-areolar complex after a partial mastectomy in patients with large breasts, we selected either an inferior or a superior pedicle after considering the tumor location and size. For patients with resected nipple-areolar complexes, a glandular reshaping procedure was performed after selecting either an inverted T-type skin incision or a vertical incision. The nipple-areolar complex was resected for most of the patients with centrally located breast cancer. In cases in which the tumor was located just under the nipple, vertical incisions were performed. In other cases, inverted T-type skin incisions were performed. To maintain symmetry between the breasts, a reduction mammaplasty of the contralateral side was performed. The mean masses of the dissected breasts were $245 \mathrm{~g}$ on the affected side and $217 \mathrm{~g}$ on the non-affected, contralateral side.

When an oncoplastic procedure was performed after a partial mastectomy, the level of aesthetic satisfaction was high and the patients' postoperative psychological stability was also improved. Additionally, the recovery period, and consequently, the hospitalization period, was short. Further, complications could be reduced by simplifying the surgery while reducing both the time that the patient remains under the influence of general anesthesia and the operation time. The cost of the overall procedure could also be reduced. The patients' aesthetic and overall satisfaction levels were high, and no noticeable complications of the procedure were noted.

Our surgical method could not be applied to all patients. When a patient has an absolute contraindication to breast-conserving surgery, our oncoplastic techniques cannot be applied. This includes diffuse malignant-appearing calcification, multicentric diseases, the inability to achieve histologically negative margins, and contraindication to radiation therapy. In such cases, mastectomy and total reconstruction are recommended.

To determine the breast reconstruction method, various fac- tors, including the mastectomy method and resection volume, size and shape of the non-affected side, distance between the nipple and tumor, length of the inframammary sulcus, donor site condition, age and health of the patient, degree of obesity, necessity of preoperative and postoperative radiation therapy or chemotherapy, and the preferences of the doctor and the patient, should be considered. In this study, various methods were used for the 35 patients. Therefore, no single oncoplastic technique could be exclusively used for all patients. Discrepancies regarding the most appropriate breast reconstruction method for each patient are inevitable. It is therefore necessary to design an appropriate surgical plan through preoperative interviews, photographs taken before surgery, and check-ups.

When performing surgical procedures for centrally located breast cancers, considering the breast size and the resection volume, an oncoplastic procedure after partial mastectomy might be a good option for breast reconstruction. Therefore, the above-described procedure can be considered a primary option for qualifying patients.

\section{REFERENCES}

1. Pezzi CM, Kukora JS, Audet IM, et al. Breast conservation surgery using nipple-areolar resection for central breast cancers. Arch Surg 2004;139:32-7.

2. Fisher ER, Gregorio R, Redmond C, et al. Pathologic findings from the National Surgical Adjuvant Breast Project (protocol no. 4). I. Observations concerning the multicentricity of mammary cancer. Cancer 1975;35:247-54.

3. Lagios MD, Gates EA, Westdahl PR, et al. A guide to the frequency of nipple involvement in breast cancer. A study of 149 consecutive mastectomies using a serial subgross and correlated radiographic technique. Am J Surg 1979;138:13542.

4. Dale PS, Giuliano AE. Nipple-areolar preservation during breast-conserving therapy for subareolar breast carcinomas. Arch Surg 1996;131:430-3.

5. Danoff BF, Pajak TF, Solin LJ, et al. Excisional biopsy, axillary node dissection and definitive radiotherapy for Stages I and II breast cancer. Int J Radiat Oncol Biol Phys 1985;11: 479-83.

6. Simmons RM, Brennan MB, Christos P, et al. Recurrence rates in patients with central or retroareolar breast cancers treated with mastectomy or lumpectomy. Am J Surg 2001; 182:325-9.

7. Horiguchi J, Koibuchi Y, Iijima K, et al. Local control by breast-conserving surgery with nipple resection. Anticancer Res 2005;25:2957-9. 
8. Huemer GM, Schrenk P, Moser F, et al. Oncoplastic techniques allow breast-conserving treatment in centrally located breast cancers. Plast Reconstr Surg 2007;120:390-8.

9. Haffty BG, Wilson LD, Smith R, et al. Subareolar breast cancer: long-term results with conservative surgery and radiation therapy. Int J Radiat Oncol Biol Phys 1995;33:53-7.

10. Fowble B, Solin LJ, Schultz DJ, et al. Breast recurrence and survival related to primary tumor location in patients undergoing conservative surgery and radiation for early-stage breast cancer. Int J Radiat Oncol Biol Phys 1992;23:933-9.

11. Alderman AK, Wilkins EG, Lowery JC, et al. Determinants of patient satisfaction in postmastectomy breast reconstruction. Plast Reconstr Surg 2000;106:769-76.

12. Veronesi U, Saccozzi R, Del Vecchio M, et al. Comparing radical mastectomy with quadrantectomy, axillary dissection, and radiotherapy in patients with small cancers of the breast. N Engl J Med 1981;305:6-11.

13. Fisher B, Bauer M, Margolese R, et al. Five-year results of a randomized clinical trial comparing total mastectomy and segmental mastectomy with or without radiation in the treatment of breast cancer. N Engl J Med 1985;312:665-73.

14. Chardot C, Carolus JM. The prevention of infections or prevention of "enlarged arm" in the treatment of breast cancer. Ann Chir Plast 1961;6:195-202.

15. Hamdi M, Van Landuyt K, de Frene B, et al. The versatility of the inter-costal artery perforator (ICAP) flaps. J Plast Reconstr Aesthet Surg 2006;59:644-52.

16. Hamdi M, Salgarello M, Barone-Adesi L, et al. Use of the thoracodorsal artery perforator (TDAP) flap with implant in breast reconstruction. Ann Plast Surg 2008;61:143-6.

17. Hunt KK, Baldwin BJ, Strom EA, et al. Feasibility of postmastectomy radiation therapy after TRAM flap breast reconstruction. Ann Surg Oncol 1997;4:377-84.

18. Maxwell GP. Iginio Tansini and the origin of the latissimus dorsi musculocutaneous flap. Plast Reconstr Surg 1980;65: 686-92.

19. Losken A, Elwood ET, Styblo TM, et al. The role of reduction mammaplasty in reconstructing partial mastectomy defects. Plast Reconstr Surg 2002;109:968-75. 